\title{
On the ambiguous treatment of the Schrödinger equation for the infinite potential well and an alternative via flat solutions: The one-dimensional case*
}

\author{
JESÚS ILDEFONSO DíAZ \\ Instituto de Matemática Interdisciplinar, Universidad Complutense de Madrid, \\ 28040 Madrid, Spain \\ E-mail: ildefonso.diaz@mat.ucm.es
}

[Received 25 July 2014 and in revised form 18 April 2015]

\begin{abstract}
An ambiguity in the mathematical treatment of the study of bound state solutions of the Schrödinger equation for infinite well type potentials (studied for the first time in a pioneering article of 1928 by G. Gamow) is pointed out. An alternative to prove a similar "localizing effect" is here offered "in terms Hardy type potentials" with the distance to the boundary as a variable. The existence of flat solutions (with zero normal derivative at the boundary) and solutions with compact support is here obtained by first time in the literature for elliptic problems for this kind of linear equations.
\end{abstract}

2010 Mathematics Subject Classification: Primary 81Q05, 35J60, 35R35, 34B15, 34C23, 35Q40.

Keywords: Schrödinger equation, infinite well potential, Hardy potentials, sublinear eigenvalue type problem, flat solution, solution with compact support.

\section{On the ambiguity and statements of an alternative treatment}

In his 1928 pioneering article Gamow [26] proved, for the first time, the tunneling effect which, among many other applications lead to the construction of the electronic microscope and the correct study of the alpha radioactivity. Most of his study was concerning with the bound states $\psi(x, t)=e^{-\mathbf{i} E t} u(x)$ of the Schrödinger equation in $\mathbb{R}^{N}, N \geqslant 1$,

$$
\mathbf{i} \hbar \frac{\partial \psi}{\partial t}=-\frac{\hbar^{2}}{2 m} \Delta \psi+V(x) \psi, \quad \text { in }(0, \infty) \times \mathbb{R}^{N},
$$

associated to the potential $V(x)$, for a single elementary particle of mass $m$ and energy $E$ (which we shall denote also by $\lambda$ ). Here $\mathbf{i}=\sqrt{-1}$ and $\hbar$ is the renormalized Planck constant.

Gamow was specially interested in the Coulomb potential

$$
V(x)=\frac{k}{|x|} \quad x \in \mathbb{R}^{N},
$$

for a suitable $k>0$, but he offered some reasons to truncate such a potential when $0<|x|<r^{\prime}$ for some $r^{\prime}>0$. Then he proposed to replace the resulting potential by a simple potential which keeps

\footnotetext{
* This work is associated with the author's participation at the "Free Boundary Problems and Related Topics", a scientific programme held at the Isaac Newton Institute of the University of Cambridge, from 6th January 2014 to 4th July 2014 , https://www. newton.ac.uk/event/frb.
} 
the main properties of the original one: in this way he proposed, it seems that for the first time in the literature, what today is usually called as the finite well potential

$$
V_{q}\left(x: R, V_{0}\right)=\left\{\begin{array}{cl}
V_{0} & \text { if } x \in(-R, R), \\
q & \text { if } x \notin(-R, R),
\end{array}\right.
$$

for a given $R>0$ and for some $V_{0}, q>0$. For the present purposes, the exact values of $m$ and $\hbar$ are not relevant and we can reformulate them in a different form. For instance assuming for simplicity $m=1$ and $\hbar=1$, we see that the spatial component $u(x)$ of the bound states must solve the stationary equation

$$
-\Delta u+V(x) u=\lambda u \quad \text { in } \mathbb{R}^{N},
$$

for a given potential $V(x)$ (possibly discontinuous).

In his article [26], Gamow consider the finite well potential by solving problem (1.3) in a weak sense: the solution was not $\mathrm{e}^{2}$ but merely $\mathrm{e}^{1}$. The notion of "solution" used by him was not explicitly mentioned in the paper but it is coherent with the notion of weak solution introduced several years later by other authors such as J. Leray, L. Sobolev and L. Schwartz.

In his paper he also made some comments on the study of the original (unbounded) Coulomb potential (1.1). Probably that was the reason why the case of the so called infinite well potential

$$
V_{\infty}\left(x: R, V_{0}\right)=\left\{\begin{array}{cl}
V_{0} & \text { if } x \in(-R, R), \\
+\infty & \text { if } x \notin(-R, R),
\end{array}\right.
$$

for some $V_{0} \in \mathbb{R}$ (which, without loss of generality, we can assume $V_{0}>0$ ) arises in the literature and started to be considered as a basic example in any text-book in Quantum Mechanics since then to our-days. In many textbooks this case is presented as a limit case of the associate finite well potential (1.2). In fact, there is an abuse of the notation in the above terminology. What is really true is that we can introduce as definition of solution $u$ of the infinite well potential problem any function $u=\lim _{q \rightarrow \infty} u_{q}$ with $u_{q}$ solution of (1.3) associated to the potential $V_{q}\left(x: R, V_{0}\right)$ given by (1.2) (see Lemma 2.1 below). It is usually claimed that $u=\lim _{q \rightarrow \infty} u_{q}$ satisfies (at least in a weak sense) equation (1.3) for the infinite well potential but, as we shall explain (see also Lemma 2.1 below) this is not correct since some other terms appear in the limit equation (which, in fact must be understood in distributional sense).

As a matter of fact, after the work by Gamow, several authors considered some generalizations of the infinite well potential corresponding to the case in which the constant value $V_{0}$ is replaced by a general function $V_{0}(x)$ leading to the potential

$$
V_{\infty}\left(x: R, V_{0}(\cdot)\right)=\left\{\begin{array}{cl}
V_{0}(x) & \text { if } x \in(-R, R), \\
+\infty & \text { if } x \notin(-R, R) .
\end{array}\right.
$$

More in general, the $N$-dimensional infinite potential well problem is defined by taking

$$
V_{\infty}\left(x: \Omega, V_{0}(\cdot)\right)=\left\{\begin{array}{cl}
V_{0}(x) & \text { if } x \in \Omega, \\
+\infty & \text { if } x \notin \Omega,
\end{array}\right.
$$

where $\Omega$ is a regular open bounded set of $\mathbb{R}^{N}$. For instance, the case of $V_{0} \in L^{1}(-R, R)$ was already considered in the 1968 monograph [31]. The more singular case in which $V_{0}(x)=\delta_{0}(x)$, the Dirac delta applied to $x=0$, related with the so called Quantum Dots, was also considered in the literature (see, e.g., Joglekar [28]). 
In contrast with the case of the tunneling effect (corresponding to the treatment of the finite well potential (1.2)) the usual study of the infinite well potential, such as it is presented in most of the textbooks, contains an ambiguity which, curiously enough, it seems unseen before: it is said in many textbooks that to solve the equation in $\mathbb{R}^{N}$ outside $\Omega$ it is necessary to impose that the solution $u(x)$ of (1.3) let $u(x) \equiv 0$ if $x \notin \Omega$ (a better justification of this fact can be given through the approximation of such potential by a sequence of truncated potentials $V_{q}$ and passing to the limit on the associated solutions $u_{q}$ as $q \rightarrow+\infty$ : see Lemma 2.1 below). Thus the study of problem (1.3) leads to solve the associated Dirichlet problem on $\Omega$

$$
D P(V, \lambda, \Omega) \begin{cases}-\Delta u+V(x) u=\lambda u & \text { in } \Omega, \\ u=0 & \text { on } \partial \Omega .\end{cases}
$$

This Dirichlet problem can be explicitly solved in many cases. For instance, for the one-dimensional $\Omega=(-R, R)$ and $V(x) \equiv V_{0}$ we get

$$
\left\{\begin{array}{l}
u_{n}(x)=C \sin \frac{n \pi}{2 R}(x+R), \\
\lambda_{n}-V_{0}=\left(\frac{\pi}{2 R}\right)^{2} n^{2}, \quad n=1,2, \ldots
\end{array}\right.
$$

In terms of the original value of the parameters $m$ and $\hbar$ and denoting again the energy by $E$ we get the discrete set of energies

$$
E_{n}:=\frac{\hbar^{2}}{2 m} \lambda_{n}
$$

(see, e.g., Strauss [34]).

The ambiguity in this mathematical treatment arises because the derivatives of the extensions of $u_{n}$ by zero on $\mathbb{R}-(-R, R)$ are discontinuous at the points $x= \pm R$, and thus such $u_{n}$ are not solutions of the equation in the whole domain $\mathbb{R}$ in the sense of distributions

$$
-\frac{\hbar^{2}}{2 m} \frac{d^{2} u_{n}}{d x^{2}}+V(x) u_{n}=E_{n} u_{n}, \quad \text { in } \mathbb{R},
$$

but of the different equation

$$
-\frac{\hbar^{2}}{2 m} \frac{d^{2} u_{n}}{d x^{2}}+V(x) u_{n}=E_{n} u_{n}+k_{n}(R) \delta_{\{R\}}-k_{n}(-R) \delta_{\{-R\}} \quad \text { in } \mathbb{R},
$$

since the second derivative develops two Dirac deltas (see Lemma 2.1 below). Here

$$
k_{n}(-R)=\frac{\hbar^{2}}{2 m} \frac{\sqrt{2}}{R^{3 / 2}} n \pi \quad \text { and } \quad k_{n}(R)=\frac{\hbar^{2}}{2 m} \frac{\sqrt{2}}{R^{3 / 2}} n \pi(-1)^{n} .
$$

The presence of such discontinuities was noticed previously in the literature (see, e.g., [25, page 140]) but, as far as we know, it seems that a careful analysis of this ambiguity, and the study of some alternative potential $V(x)$ preventing it, was not considered before.

Besides pointing out such ambiguity (see a more detailed presentation in Lemma 2.1 below), the main goal of this paper is to present a set of results offering some kind of alternative. In particular, here we shall deal merely with nonnegative solutions $u \geqslant 0$ of $D P(V, \lambda, \Omega)$, and in fact in the one-dimensional case, $\Omega=(-R, R)$. Our purpose is to give an answer to the following inverse 
free boundary problem: can we find a potential $V(x)$ and some values of the energy $\lambda$ such that the solution of $-\frac{d^{2} u}{d x^{2}}+V(x) u=\lambda u$ in $\mathbb{R}$, gives rise to a free boundary given by $\partial \Omega$ in the sense that $u \equiv 0$ on $\mathbb{R} \backslash \Omega$ and $\frac{d u}{d x}( \pm R)=0$ ?

We comment now that the case of higher dimensions will be the object of a separated work by this author [16] and that the case of nodal solutions (i.e, changing sign on $\Omega$ ) for the associated semilinear problem was the main object of the paper Díaz and Hernández [20].

It is useful to introduce the following notation (already used in the literature):

DEFINITION 1.1 We say that a function $u \in H_{0}^{1}(\Omega)$ is a (positive) "flat solution" of problem $D P(V, \lambda, \Omega)$ if $u$ satisfies $D P(V, \lambda, \Omega), u>0$ and $\frac{d u}{d x}( \pm R)=0$.

We point out that this type of special solutions was called previously by other authors as "free boundary solutions" (see, e.g., [29]). Nevertheless in our opinion the use of the expression "free boundary" may be misleading: such terminology is more adequate in the context where the equation is set in the whole real line (as in (1.3)) and not in a given bounded interval.

Our main result of this paper (improving the presentation made by the author in a series of lectures [15]) is the following:

THEOREM 1.2 Let $\Omega=(-R, R)$ and let $V \in L_{\text {loc }}^{1}(\Omega)$ be such that

$$
\frac{\underline{C}}{d(x, \partial \Omega)^{\alpha}} \leqslant V(x) \leqslant \frac{\bar{C}}{d(x, \partial \Omega)^{\alpha}} \quad \text { a.e. } x \in \Omega=(-R, R),
$$

for some $\alpha \in[0,2]$ and some $\bar{C}>\underline{C}>0$. Then:

1. If $\alpha \in[0,2)$ then, for any $\lambda \geqslant 0$, no positive solution of $D P(V, \lambda, \Omega)$ may be a flat solution.

2. If $\alpha=2$, for any value of $\bar{C}$ and $\underline{C}$, there exists $\lambda^{\#}=\lambda^{\#}(R) \geqslant\left(\frac{\pi}{2 R}\right)^{2}$ such that problem $D P\left(V, \lambda^{\#}, \Omega\right)$ has a nonnegative solution $u^{\#}$.

3. If $\alpha=2$, given $u^{\#}$ nonnegative solution of $D P\left(V, \lambda^{\#}, \Omega\right)$ as in Part 2 , there exists $m^{\#} \in[0,1)$ and there exists two positive constants $C_{*}\left(m^{\#}\right)<C^{*}\left(m^{\#}\right)$ such that if

$$
C_{*} \leqslant \underline{C} \leqslant \bar{C}<C^{*}
$$

then $u^{\#}$ is a non-degenerate positive flat solution in the sense that

$$
\underline{K} d(x, \partial \Omega)^{2 /\left(1-m^{\#}\right)} \leqslant u^{\#}(x) \leqslant \bar{K} d(x, \partial \Omega)^{2 /\left(1-m^{\#}\right)} \quad \text { for any } x \in \bar{\Omega},
$$

for some constants $\bar{K}\left(m^{\#}\right)>\underline{K}\left(m^{\#}\right)>0$. Moreover $u^{\#} \in \mathrm{e}^{2 /\left(1-m^{\#}\right)}(\bar{\Omega})$.

COROllary 1.3 Let $\Omega=(-R, R)$ and $V_{\infty}\left(x: R, V_{0}(\cdot)\right)$ with $V_{0}(\cdot)$ satisfying (1.7) and (1.8) with $\alpha=2$. Then there exists $\lambda^{\#}>0$ such that the Schrödinger equation (1.3), with $N=1$, admits a solution $u \in \mathrm{C}^{2 /\left(1-m^{\#}\right)}(\mathbb{R})$ satisfying (1.9), for suitable $m^{\#} \in(0,1), \bar{K}>\underline{K}>0$, and such that $u \equiv 0$ on $\mathbb{R} \backslash \Omega$.

As far as we know, Theorem 1.2 is the first result in the literature showing the existence of a flat solution for a linear elliptic problem. We recall that the first result in the literature on solutions with compact support for elliptic problems was raised in the works Brezis and Stampacchia [12] and [13] related to an obstacle problem formulated for the study of subsonic flows. Later the existence of solutions with compact support was extended to other semilinear (sublinear) problems in Brezis [11] 
and Benilan, Brezis and Crandall [10]. Many other results for nonlinear problems can be found, for instance, in the monograph [14]. Of course the existence of the flat solution is only possible when the strong maximum principle cannot be applied (see e.g. [32], [35] and [33]).

REMARK 1.4 When $\bar{C}=\underline{C}$ potentials $V(x)$ satisfying (1.7), with $\alpha=2$, are called "Hardy type potentials" on the distance to the boundary variable. There is a long literature dealing with problems involving such potentials. We emphasize that here we are considering the so called "absorption case" and that, in contrast with other authors considering the formation of a free boundary (see, e.g., [3]), the main problem under consideration in this paper is linear. Moreover, we point out that the solution $u^{\#}$ mentioned in Part 2 of the above theorem exists for any value of the constants $\underline{C}$ and $\bar{C}$ : so, if , for instance we have two potentials $V_{i}(x)$, with $i=1,2$, satisfying (1.7) with $\alpha=2$ and some constants $\underline{C}_{i} \leqslant \bar{C}_{i}$ such that $\bar{C}_{1}<\underline{C}_{2}$ then the application of Part 2 implies that the corresponding eigenvalues $\lambda_{i}^{\#}$ and nonnegative eigenfunctions $u_{i}^{\#}$ of problem $D P\left(V_{i}, \lambda^{\#}, \Omega\right)$ verify that $u_{1}^{\#} \neq u_{2}^{\#}$. In consequence, in Part 3 of Theorem 1.2 we would have the similar conclusion for different values $m_{i}^{\#} \in[0,1)$ and positive constants $C_{*}\left(m_{i}^{\#}\right)<C^{*}\left(m_{i}^{\#}\right)$ for $i=1,2$.

After the above comments on the literature on solutions with compact support for nonlinear problems perhaps it is not too strange to say that we use here some auxiliary nonlinear problem giving rise to positive non-degenerate flat solutions in order to prove Theorem 1.2. To be more precise, we shall start considering the nonlinear eigenvalue type problem

$$
P\left(R, m, V_{0}, \lambda\right) \equiv\left\{\begin{array}{l}
-\frac{d^{2} v}{d x^{2}}+V_{0} v^{m}=\lambda v, \quad v \geqslant 0 \text { in }(-R, R), \\
v( \pm R)=0,
\end{array}\right.
$$

for a given $V_{0}>0$ and $m \in(0,1)$. We shall prove:

Proposition 1.5 For any $\lambda \geqslant\left(\frac{\pi}{2 R}\right)^{2}$ there exists a unique nonnegative solution $v_{m}$ of $P\left(R, m, V_{0}, \lambda\right)$. Moreover, there exists a $\lambda^{*}(m)>\left(\frac{\pi}{2 R}\right)^{2}$ such that:

(i) If $\lambda \geqslant \lambda^{*}(m)$ then

$$
v_{m}(x) \leqslant \bar{K} d(x, \partial \Omega)^{2 /(1-m)} \text { for any } x \in \bar{\Omega}=[-R, R],
$$

for some constant $\bar{K}$. In particular $\frac{d v_{m}}{d x}( \pm R)=0$ and $v_{m}$ is a flat solution.

(ii) If $\lambda \leqslant \lambda^{*}(m)$ then

$$
\underline{K} d(x, \partial \Omega)^{2 /(1-m)} \leqslant v_{m}(x) \text { for any } x \in \bar{\Omega}=[-R, R],
$$

for some constant $\underline{K}$. In particular $v_{m}>0$ in $\Omega$ is a non-degenerate solution. (iii) If $\lambda=\lambda^{*}(m)$ inequalities (1.10) and (1.11) hold for some $\bar{K}>\underline{K}>0$.

REMARK 1.6 It is possible to get many variants of the above mentioned results. For instance the spatial interval $\Omega=(-R, R)$ can be replaced by any other bounded interval not necessarily symmetric or even by an unbounded interval of the form $\Omega=(0,+\infty)$. In this last case the assumptions on the potential $V(x)$ are

$$
\frac{\underline{C}}{x^{2}} \leqslant V(x) \leqslant \frac{\bar{C}}{x^{2}} \text { for any } x \in\left(0, x_{0}\right) \text { for some } x_{0}>0
$$


and

$$
\underline{C} \leqslant \lim _{x \rightarrow+\infty} \inf V(x) x^{2} \leqslant \lim _{x \rightarrow+\infty} \sup V(x) x^{2} \leqslant \bar{C} .
$$

Notice that under the above condition the spectrum is still countable (see, e.g., [25]). The study of flat solutions at $x=0$ under condition (1.12) when assumption (1.13) fails (as for instance for the "effective potential" associated to the Yukawa potential: also called "screened Coulomb potential"

$$
W(r)=\frac{L_{0}}{\mu r^{2}}+\frac{k}{r} e^{-\frac{r}{a}}
$$

with $L_{0}$ the angular momentum, $\mu$ the reduced mass and $k \in \mathbb{R}, a>0$ some parameters) can be considered by means of the local techniques presented in [16].

Concerning the case of nodal solutions of the semilinear problem $P\left(R, m, V_{0}, \lambda\right)$ constructed in [20] we have:

COROLLARY 1.7 Estimates similar to the ones given by (1.10) and (1.11) also apply to $\left|u_{\lambda_{n}^{*}(m)}\right|$ with $u_{\lambda_{n}^{*}(m)}$ the nodal solutions of the semilinear problem $P\left(R, m, V_{0}, \lambda\right)$ corresponding to suitable values $\lambda_{n}^{*}(m)$ of the parameter $\lambda$ in branches bifurcating at the infinity from the simple eigenvalues $\lambda_{n}$ for any $n \in \mathbb{N}$.

We recall that the sentence "bifurcating at the infinity from the simple eigenvalues $\lambda_{n}$ " simply means that the curves $\left(\lambda, u_{\lambda}\right)$, with $u_{\lambda}$ solution of $P\left(R, m, V_{0}, \lambda\right)$, are such that $l i m_{\lambda} \lambda_{n} u_{\lambda}=u_{\lambda n}$ with $u_{\lambda n}$ eigenfunctions of the linear problem $D P(0, \lambda, \Omega)$.

As a particular consequence of Corollaries 1.3 and 1.7 it is possible to offer a correct alternative to the "localizing" process suggested by Gamow in his paper [26].

COROLlaRY 1.8 For any $R>0, n \in \mathbb{N}$ and $m \in(0,1)$ there exists a countable set of values of the parameter $\lambda=\lambda_{n}^{*}(m)$ (in branches bifurcating at the infinity from the simple eigenvalues $\lambda_{n}$, of the linear problem $(D P(0, \lambda, \Omega))$, and there exists a countable set of infinite well type potentials $V_{n, m}(x)=V_{\infty}\left(x: R, V_{0, n, m}().\right)$ such that the associated Schrödinger equation

$$
-\frac{d^{2} u}{d x^{2}}+V_{n, m}(x) u=\lambda_{n}^{*}(m) u \text { in } \mathbb{R},
$$

admits a solution $u_{n, m} \in C^{\frac{2}{1-m}}(\mathbb{R})$, changing sign $n$-times, such that $u_{n, m}(x)=0$ for any $x \notin$ $(-R, R)$ (and in particular $u_{n, m}^{\prime}( \pm R)=0$ ). Moreover $V_{\infty}\left(x: R, V_{0, n, m}(x)\right) u_{n, m}(x)=0$ for any $x \notin(-R, R)$ (i.e. no Dirac delta is generated on the boundaries $x= \pm R$ ).

The proof of Corollary 1.8 holds now simply by taking

$$
V_{\infty}\left(x: R, V_{0, n, m}(x)\right)= \begin{cases}V_{0}\left|v_{\lambda_{n}^{*}(m)}(x)\right|^{m-1} & \text { if } x \in(-R, R), \\ +\infty & \text { if } x \notin(-R, R),\end{cases}
$$

where $v_{\lambda_{n}^{*}(m)}$ is the solution of the semilinear problem $P\left(R, m, V_{0}, \lambda\right)$ associated to $\lambda=\lambda_{n}^{*}(m)$. Some comments on the regularity $u_{n, m} \in \mathrm{C}^{\frac{2}{1-m}}(\mathbb{R})$ are offered in Remark 2.1 below. In fact, Theorem 1.2 proves that there are many other potentials leading to the "localizing" process suggested by Gamow. 
We shall end the paper by proving, in Section 2, that the existence of flat solutions also holds for other different linear problems in the presence of absorption potentials of Hardy type. More precisely, we consider the nonhomogeneous problem

$$
D P(V, f, R)\left\{\begin{array}{l}
-\Delta u+V(x) u=f(x) \\
u( \pm R)=0 .
\end{array} \quad \text { in }(-R, R),\right.
$$

We shall prove:

THEOREM 1.9 Let $\Omega=(-R, R)$ and let $V \in L_{\text {loc }}^{1}(\Omega)$ satisfying (1.7) for $\alpha=2$. Let $f \in L^{1}(\Omega)$, $f(x) \geqslant 0$, a.e. $x \in(-R, R)$ such that

$$
f(x) \leqslant \bar{K} d(x, \partial \Omega)^{(1+m) /(1-m)} \text { a.e. } x \in(-R, R),
$$

for some suitable $m \in(0,1)$ and $\bar{K}>0$. Then the (unique) weak solution $u$ of $D P(V, f, R)$ satisfies

$$
0 \leqslant u(x) \leqslant K d(x, \partial \Omega)^{2 /(1-m)} \text { for any } x \in \bar{\Omega}=[-R, R]
$$

for some constant $K$. In particular $\frac{d u}{d x}( \pm R)=0$.

\section{Proofs and additional remarks}

Let us start by presenting now the details about the convergence of solutions $u_{q}$ of the problem in the whole space (1.3) when the potential $V$ is replaced by a family of finite well potentials $V_{q}\left(x: R, V_{0}\right)$ (for a given $R>0$ and for some $V_{0}$ ) and $q \rightarrow+\infty$. This fact is developed in many textbooks but usually the convergence is not well indicated in the sense that it is not indicated the functional space in which the convergence of solutions $u_{q}$ takes place.

Lemma 2.1 Given $q>0$ and $V_{q}\left(x: R, V_{0}\right)$ defined by (1.2) problem (1.3), with $N=1$, has a numerable sequence of eigenvalues $\lambda_{n}(q)$ and eigenfunctions $u_{q, n}(x)$ (renormalized such that $\left.\left\|u_{q, n}\right\|_{L^{2}(\mathbb{R})}=1\right)$. Moreover, as $q \rightarrow+\infty$,

$$
\lambda_{n}(q) \rightarrow\left(\frac{\pi}{2 R}\right)^{2} n^{2}, \quad \text { with } n \in \mathbb{N},
$$

and $u_{q, n} \rightarrow u_{n}$ weakly in $H^{1}(\mathbb{R})$, with $u_{n}$ given by (1.5) and $u_{n}$ extended by zero on $\mathbb{R}-(-R, R)$. Finally, $\left(u_{n}\right)_{x x}$ generate two family of Dirac deltas (depending on $n \in \mathbb{N}$ ): one at $x=R$ and the other at $x=-R$.

Proof. We shall follow here some of the computations made in [30] (see Section 4.7). Without lost of generality we can assume $V_{0} \equiv 0$. The function $u_{q}$ can be written as

$$
u_{q}(x)=\left\{\begin{array}{cr}
A e^{\beta x}+B e^{-\beta x} & x<-R, \\
C e^{i \alpha x}+D e^{-i \alpha x} & -R<x<R, \\
F e^{\beta x}+G e^{-\beta x} & R<x,
\end{array}\right.
$$

for suitable constants $A, B, C, D, F, G$ and

$$
\alpha=\sqrt{\lambda}, \beta=\sqrt{q-\lambda} .
$$


Since we search solutions $u_{q}$ in $H^{2}(\mathbb{R})$ we get two kinds of necessary conditions: i) the integrability of $\left|u_{q}\right|^{2},\left|\left(u_{q}\right)_{x}\right|^{2}$ and $\left|\left(u_{q}\right)_{x x}\right|^{2}$ on $\mathbb{R} \backslash(-R, R)$ (which implies conditions $B=F=0$ ), and ii) the continuity of $u_{q}$ and $\left(u_{q}\right)_{x}$ at the matching points $x= \pm R$ (which implies the conditions $C= \pm D$ : as a matter of fact we also deduce that $C=D$ implies $A=G$ and that $C=-D$ implies $A=-G$, i.e. the solutions $u_{q}(x)$ exhibit the even or odd symmetry, or parity, with respect to $x$ ). Moreover the identity in the differential equation leads to the following conditions on $\lambda$ :

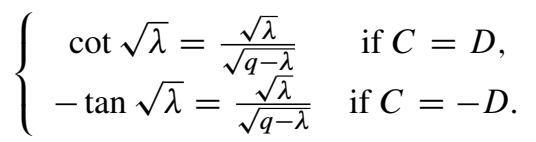

It is not too difficult to show ([30]) that, given $q \in(0,+\infty)$, the above transcendent equations have a discrete set of solutions $\lambda_{n}(q)$ and that

$$
\lambda_{n}(q) \rightarrow\left(\frac{\pi}{2 R}\right)^{2} n^{2}, \quad \text { as } q \rightarrow+\infty, \text { with } n \in \mathbb{N} .
$$

Moreover, the above set of solutions $u_{q, n}(x)$ is renormalized such that

$$
\left\|u_{q, n}\right\|_{L^{2}(\mathbb{R})}=1
$$

(since we want that the associated wave function represents a probability density). Then, by multiplying the differential equation by $u_{q, n}$, using that $V_{q}\left(x: R, V_{0}\right) \geqslant 0$ and integrating we conclude that $\left\|u_{q, n}\right\|_{H^{1}(\mathbb{R})}$ is uniformly bounded (and thus $\left\|u_{q, n}\right\|_{L^{p}(\mathbb{R})}$ is uniformly bounded too for any $p \in[1,+\infty])$. Then $u_{q, n} \rightarrow u_{n}$ weakly in $H^{1}(\mathbb{R})$ and strongly in $L^{2}(I)$ for any bounded open interval $I \subset \mathbb{R}$. From the expression (2.1) we deduce that $u_{q, n}(x) \rightarrow 0$ if $x \notin(-R, R)$ and that $u_{q, n}(x) \rightarrow C_{n}^{*} \sin \frac{n \pi}{2 R}(x+R)$ if $x \in(-R, R)$, as $q \rightarrow+\infty$, for a suitable constant $C_{n}^{*}$ such that

$$
\int_{-R}^{R}\left(C_{n}^{*}\right)^{2}\left(\sin \frac{n \pi}{2 R}(x+R)\right)^{2} d x=1 .
$$

In other words, $\lambda_{n}(q) \rightarrow \lambda_{n}=\left(\frac{\pi}{2 R}\right)^{2} n^{2}$ and $u_{n}$ is the function given in (1.5). Obviously $\left(u_{q, n}\right)_{x x}$ is discontinuous (although it belongs to $L^{2}(\mathbb{R})$ ) since $V_{q}\left(x: R, V_{0}\right) u_{q, n}(x)$ is a discontinuous function. Moreover we have $V_{q}\left(x: R, V_{0}\right) u_{q, n}(x) \rightarrow 0$ if $x \notin(-R, R)$. Thus $u_{n} \notin H^{2}(\mathbb{R})$ since the first directional derivatives verify that $\left(u_{n}\right)_{x}(-R+) \neq 0$ and $\left(u_{n}\right)_{x}(R-) \neq 0$. Then, as indicated in the introduction, there are two Dirac deltas generated by $\left(u_{n}\right)_{x x}$ : one at $x=R$ and the other at $x=-R$ (the ones appearing in the equation (1.6) when we assume $\hbar^{2} / 2 m=1$ ).

Now we pass to consider the proof of Theorem 1.2. As already mentioned the key point of it will be the set of estimates stated in Proposition 1.5 for the solutions of the auxiliary nonlinear problem $P\left(R, m, V_{0}, \lambda\right)$. To prove such estimates we shall use some suitable transformations and plane phase methods of ordinary differential equations. These type of arguments were used in [18] (extended in [22] to the case of $m \in(-1,1))$ to a variation of the equation $P\left(R, m, V_{0}, \lambda\right)$. They have the advantage of providing a complete description of the solution set for $P\left(R, m, V_{0}, \lambda\right)$, something that cannot be expected for the $N$-dimensional problem. The existence of a branch of positive solutions for a bounded interval of the parameter, $\lambda \in\left(\lambda_{1}, \lambda^{*}(m)\right)$ was proven in [20, Theorem 1]. We recall 
that $\lambda_{1}=\left(\frac{\pi}{2 R}\right)^{2}$ is the first eigenvalue of the linearized problem $D P(0, \lambda, \Omega)$

$$
\begin{cases}-\frac{d^{2} u}{d x^{2}}=\lambda u & \text { in } \Omega \\ u=0 & \text { on } \partial \Omega\end{cases}
$$

where $\Omega=(-R, R)$. Here $\lambda^{*}(m)$ is a certain value of the parameter whose exact definition depends crucially on the main assumption $m \in(0,1)$ :

$$
\lambda^{*}(m)=\frac{1}{2 R^{2}}\left(\int_{0}^{(2 /(1+m))^{1 /(1-m)}} \frac{d r}{(-F(r))^{1 / 2}}\right)^{2}
$$

with

$$
F(r)=\frac{r^{2}}{2}-\frac{r^{m+1}}{m+1}
$$

It is shown in [20, Theorem 1] that the (unique) positive solution for $\lambda=\lambda^{*}(\mathrm{~m})$ has a peculiar behaviour near the boundary: it is a "flat positive solution" in the sense that $u>0$ in $\Omega$ and

$$
\frac{\partial u}{\partial n}(x)=0 \text { on } \partial \Omega
$$

The associated solution $u_{\lambda^{*}(m), V_{0}}$ (when extended by zero to the whole real line $\mathbb{R}$ ) gives rise to a continuum of nonnegative solutions $u_{\lambda, V_{0}}$ for any $\lambda>\lambda^{*}(m)$ through a double rescaling (in amplitude and in the argument of application). This type of solutions have compact support in the sense that

$$
\operatorname{support}\left(u_{\lambda, V_{0}}\right) \subsetneq \Omega \text {. }
$$

In [20, Theorem 2] we show a qualitatively similar result for the branches of nodal solutions changing sign a finite number of times and emanating from the infinity from the simple eigenvalues $\lambda_{n}$, for $n>1$, of the linear problem (2.2). The main novelties of Proposition 1.5 are the estimates (1.10) and (1.11).

Proof of Proposition 1.5. Let $F(r)$ be given by (2.4) and let

$$
r_{F}=(2 /(1+m))^{1 /(1-m)} .
$$

For $\mu \in\left[r_{F},+\infty\right)$ we define

$$
\gamma(\mu):=\frac{1}{\sqrt{2}} \int_{0}^{\mu} \frac{d r}{(F(\mu)-F(r))^{1 / 2}} .
$$

It is shown in [20, Theorem 1] that the mapping $\gamma:\left[r_{F},+\infty\right) \rightarrow \mathbb{R}$ has the following properties: (i) $\gamma \in C\left[r_{F}, \infty\right) \cap C^{1}\left(r_{F}, \infty\right)$; (ii) $\gamma^{\prime}(\mu) \rightarrow-\infty$ as $\mu \downarrow r_{F}$; (iii) For any $\mu>r_{F} \gamma^{\prime}(\mu)<0$, (iv) $\lim _{\mu \rightarrow+\infty} \gamma(\mu)=\frac{\pi}{2}$. Moreover, it was also shown there that if $\lambda^{*}(m)$ is given by (2.3) then we know that:

(a) if $\lambda \in\left(0,\left(\frac{\pi}{2 R}\right)^{2}\right)$ there is no positive solution, 
(b) if $\lambda \in\left(\left(\frac{\pi}{2 R}\right)^{2}, \lambda^{*}(m)\right)$ there is a unique positive solution $v_{\lambda, V_{0}}$. Moreover $\frac{\partial v_{\lambda}, V_{0}}{\partial n}( \pm R)<0$ and $\left\|v_{\lambda, V_{0}}\right\|_{L^{\infty}(-R . R)}=\left(\frac{V_{0}}{\lambda}\right)^{\frac{1}{1-m}} \gamma^{-1}(\sqrt{\lambda} R)$,

(c) if $\lambda=\lambda^{*}(m)$ there is only one positive solution $v_{\lambda *(m), V_{0}}$. Moreover $v_{\lambda^{*}(m), V_{0}}^{\prime}( \pm R)=0$

$$
\left\|v_{\lambda^{*}(m), V_{0}}\right\|_{L^{\infty}(-R, R)}=\left(\frac{2 V_{0}}{\lambda^{*}(m)(1+m)}\right)^{\frac{1}{1-m}},
$$

(d) if $\lambda>\lambda^{*}(m)$, there is a family of nonnegative solutions which is generated by extending by zero the function $v_{\lambda *(m), V_{0}}$ outside $(-R, R)$ (and which we label again as $\left.v_{\lambda *(m), V_{0}}\right)$. In particular, if $\lambda=\lambda^{*}(m) \omega$ with $\omega>1$ we have a family $S_{1}(\lambda)$ of compact support nonnegative solutions with connected support defined by

$$
v_{\lambda, V_{0}}(x)=\frac{1}{\omega^{\frac{1}{1-m}}} v_{\lambda *(m), V_{0}}(\sqrt{\omega} x-z)
$$

where the shifting argument $z$ is arbitrary among the points $z \in(-R, R)$ such that support $v_{\lambda, V_{0}}(\cdot) \subset(-R, R)$. Moreover, for $\lambda>\lambda^{*}(m)$ large enough we can build, similarly, a subset of $S_{j}(\lambda)$ of compact support nonnegative solutions with the support formed by $j$-components, with $j \in\{1,2, \ldots, N\}$, for some suitable $N=N(\lambda)$ and then the set of nontrivial and nonnegative solutions of $P(\lambda)$ is formed by $S(\lambda)=\cup_{j=1}^{N} S_{j}(\lambda)$. In any case these solutions satisfy that

$$
\left\|v_{\lambda, V_{0}}\right\|_{L^{\infty}(-R, R)}=\frac{1}{\omega^{\frac{1}{1-m}}}\left\|v_{\lambda^{*}(m), V_{0}}\right\|_{L^{\infty}(-R, R)}=\frac{1}{\omega^{\frac{1}{1-m}}}\left(\frac{2 V_{0}}{\lambda^{*}(m)(1+m)}\right)^{\frac{1}{1-m}},
$$

for any $\omega=\lambda / \lambda^{*}(m)>1$.

In order to prove the estimates (1.10) and (1.11) we need to reconstruct some of the arguments of the proof of Theorem 1 of [20]. We make the change of variables

$$
v_{\lambda, V_{0}}(x)=\left(\frac{V_{0}}{\lambda}\right)^{\frac{1}{1-m}} v(\sqrt{\lambda} x)
$$

where $v$ is now the solution of the renormalized problem

$$
P(L)\left\{\begin{array}{l}
-v^{\prime \prime}=f(v) \\
v( \pm L)=0
\end{array} \quad \text { in }(-L, L),\right.
$$

with $f(v)=v-v^{m}$ and $L=\sqrt{\lambda} R$. We introduce $F(r)$ given by (2.4) and note that $F(s)<0$ if $0<s<r_{F}$ and $F(s)>0$ for $s>r_{F}$.

For $\mu>r_{F}$ we define the mapping $\gamma:\left[r_{F},+\infty\right) \rightarrow \mathbb{R}$ given by (2.6). Now we use the following fact whose proof is exactly as in [18] and [22]: a function $v$ is a positive solution of problem $P(L)$ if and only if

$$
\frac{1}{\sqrt{2}} \int_{v(x)}^{\mu} \frac{d r}{(F(\mu)-F(r))^{1 / 2}}=|x|, \text { for }|x| \leqslant L,
$$


where $\mu:=\|v\|_{L^{\infty}}$ (such that $\mu \in\left(r_{F}, \infty\right)$ ) and $L>0$ are related by the equation $\gamma(\mu)=L$. In particular, if $\mu>r_{F}$ we get that

$$
v^{\prime}( \pm L)=\mp \sqrt{2 F(\mu)} .
$$

Thus

$$
\left|v^{\prime}( \pm L)\right|=|\sqrt{2 F(\mu)}|>0,
$$

which proves Part (ii) of the Proposition 1.5 for the case of $\lambda<\lambda^{*}(m)$ since we know that it corresponds to the case in which the transformed function $v$ by the change of variables (2.8) has a maximum $\mu$ such that $\mu>r_{F}$. In the case of $\lambda=\lambda^{*}(m)$ the associated function $v$ is such that $\mu=r_{F}$ and in consequence $v^{\prime}( \pm L)=0$. Moreover, since

$$
\frac{1}{1+m} r^{1+m} \geqslant \frac{1}{1+m} r^{1+m}-\frac{1}{2} r^{2} \geqslant \frac{(1-m)}{2(1+m)} r^{1+m} \quad \text { for } r \in(0,1),
$$

we get that there exist two positive constants $\underline{M}<\bar{M}$ such that

$$
\underline{M} \tau^{\frac{1-m}{2}} \leqslant \frac{1}{\sqrt{2}} \int_{0}^{\tau} \frac{d r}{\sqrt{-F(r)}} \leqslant \bar{M} \tau^{\frac{1-m}{2}}
$$

for any $\tau \in(0,1)$ which leads to conclusion (iii) of Proposition 1.5 (and obviously also ii) for $\left.\lambda=\lambda^{*}(m)\right)$.

Finally, since we know that for $\lambda>\lambda^{*}(m)$ the nonnegative solutions are generated extending by zero the function $v_{\lambda^{*}(m), V_{0}}$ outside $(-R, R)$ we get Part (i) of Proposition 1.5 thanks to the estimate (2.11).

REMARK 2.2 Note that estimate (1.10) proves that if $\lambda \geqslant \lambda^{*}(m)$ then the solution $v$ of $P\left(R, m, V_{0}, \lambda\right)$ is more regular than the usual definition of classical solution since $v \in$ $\mathrm{C}^{\frac{2}{1-m}}([-R, R])$. As a matter of fact, given $L>0$ we can produce $\mathrm{C}^{\infty}$ functions with compact support, contained in the open interval $(-L, L)$, and being solutions of problem $P(L)$ when we take as a function $f(v)$ a variation of the function $f$ arising in the above proof. Indeed, by taking

$$
F(v)=\frac{v^{2}}{2}-v^{2}(\ln v)^{2 a}
$$

for a given $a>1$, we get that $f(v)=F^{\prime}(v)$ satisfies that

$$
f(v) \leqslant v-v^{m}
$$

for any $m \in(0,1)$ but still

$$
\int_{0}^{\mu} \frac{d r}{\sqrt{-F(r)}}<+\infty
$$

for any $\mu>0$. It is a routine matter to check that

$$
\underline{M} \tau^{\frac{1-m}{2}} \leqslant \frac{1}{\sqrt{2}} \int_{0}^{\tau} \frac{d r}{\sqrt{-F(r)}} \leqslant \bar{M} \tau^{\frac{1-m}{2}}
$$

for any $m \in(0,1)$ and thus, by taking the solution of maximum $\mu=r_{F}$ with $r_{F}$ the first positive zero of $F$ (i.e. such that $F(s)<0$ if $0<s<r_{F}$ and $F(s)>0$ for $s>r_{F}$ ) we get that the 
estimate (1.10) remains valid for any $m \in(0,1)$. Thus $v \in \mathrm{C}^{\frac{2}{1-m}}([-R, R])$ for any $m \in(0,1)$. This gives an answer (for the one-dimensional case) to a question raised to the author by Jean-Michel Coron during his visit to Madrid on May 2014.

REMARK 2.3 The constants $\underline{M}<\bar{M}$ arising in (2.11), in the proof of Proposition 1.5, can be estimated in a sharper way. Indeed, by l'Hôpital rule we have

$$
\lim _{\tau \searrow 0} \frac{\frac{1}{\sqrt{2}} \int_{0}^{\tau} \frac{d r}{\sqrt{-F(r)}}}{\tau^{\frac{1-m}{2}}}=\frac{\sqrt{2}}{2(1-m)} \lim _{\tau \searrow 0} \frac{\tau^{\frac{m+1}{2}}}{\sqrt{-F(r)}}=\frac{\sqrt{2}}{2(1-m) \sqrt{m+1}}:=C(m)
$$

Then estimates (2.11) holds with

$$
\underline{M}=(C(m)-\theta), \bar{M}=(C(m)-\theta) \text { for some } \theta>0 .
$$

As a matter of fact, since we have

$$
\bar{M} v(L-y)^{\frac{1-m}{2}} \geqslant \gamma(v(L-y))=y \quad \text { for any } y \in[0, L]
$$

(and analogously with the other estimate), we get the estimates of the statement of Proposition 1.5 with

$$
\bar{K}=\frac{V_{0}^{\frac{1}{1-m}}}{\lambda^{*}(m)^{\frac{m}{1-m}}(C(m)-\theta)^{\frac{2}{1-m}}}, \quad \underline{K}=\frac{V_{0}^{\frac{1}{1-m}}}{\lambda^{*}(m)^{\frac{m}{1-m}}(C(m)+\theta)^{\frac{2}{1-m}}} .
$$

Note that, curiously, if $v_{\lambda^{*}(m)}\left(x: V_{0}\right)$ is the flat solution of $P\left(R, m, V_{0}, \lambda^{*}(m)\right)$ then

$$
\frac{V_{0}}{\left|v_{\lambda^{*}(m)}(x)\right|^{1-m}} \leqslant \frac{V_{0}}{(K)^{1-m}} \frac{1}{d(x, \partial \Omega)^{2}}=\lambda^{*}(m)^{m}(C(m)-\theta)^{2} \frac{1}{d(x, \partial \Omega)^{2}},
$$

and

$$
\frac{V_{0}}{\left|v_{\lambda^{*}(m)}(x)\right|^{1-m}} \geqslant \frac{V_{0}}{(\bar{K})^{1-m}} \frac{1}{d(x, \partial \Omega)^{2}}=\lambda^{*}(m)^{m}(C(m)+\theta)^{2} \frac{1}{d(x, \partial \Omega)^{2}},
$$

i.e. with estimates independent of $V_{0}$.

Now we can prove the main result of this paper:

Proof of Theorem 1.2. Part 1 holds since if $\alpha<2$ then $\frac{d v}{d x}(-R)>0$ and $\frac{d v}{d x}(R)<0$. The proof is an easy adaptation of the Höpf strong maximum principle, see, e.g., [32], [9] and [27].

In order to prove Part 2 we shall start by arguing as in the proof of Theorem 3.2 of [23] (see also some further results in [21]). For any $h \in L^{2}(\Omega)$ (recall that $\Omega=(-R, R)$ ) we define the operator $T h=z \in H_{0}^{1}(\Omega)$ solution of the linear problem

$$
\left\{\begin{array}{lr}
-\frac{d^{2} z}{d x^{2}}+V(x) z=h & \text { in } \Omega \\
z=0 & \text { on } \partial \Omega
\end{array}\right.
$$

This operator is well defined since problem (2.15) has a unique (weak) solution $z \in H_{0}^{1}(\Omega)$. This follows from applying the Lax-Milgram Lemma to the associated bilinear form in $H_{0}^{1}(\Omega)$

$$
a(u, v)=\int_{\Omega} \frac{d u}{d x} \frac{d v}{d x} \mathrm{~d} x+\int_{\Omega} V(x) u v \mathrm{~d} x
$$


which is well-defined, continuous and coercive. Indeed, taking into account that

$$
V(x) \leqslant \frac{\bar{C}}{d(x, \partial \Omega)^{\alpha}} \quad \text { a.e. } x \in \Omega
$$

(thanks to assumption (1.7)), Hardy's inequality implies that

$$
\frac{1}{\bar{C}} \int_{\Omega} V(x) u^{2} \mathrm{~d} x \leqslant \int_{\Omega} \frac{u^{2}}{d(x)^{2}} \mathrm{~d} x \leqslant k \int_{\Omega}\left|\frac{d u}{d x}\right|^{2} \mathrm{~d} x
$$

for some suitable constant $k=k(\Omega)$ and then

$$
a(u, u) \leqslant C\|u\|_{H_{0}^{1}(\Omega)}^{2}
$$

for some $C>0$, which implies that $a$ is continuous (the coerciveness of $a$ is a routine matter). Thus, for any $h \in L^{2}(\Omega)$, there exists a unique $T h \in H_{0}^{1}(\Omega)$ solution of the above equation and it is easy to see that the composition with the (compact) embedding $H_{0}^{1}(\Omega) \subset L^{2}(\Omega)$ is a self-adjoint compact linear operator $\widetilde{T}=i \circ T: L^{2}(\Omega) \rightarrow L^{2}(\Omega)$ for which we obtain in the usual way a sequence of eigenvalues $v_{n} \rightarrow+\infty$. If we call $\lambda^{\#}=v_{1}$ then, by well-known results we know that $\lambda^{\#}>0$. In fact, since $V(x) \geqslant 0$, we know that $\lambda^{\#}>\lambda_{1}(R)=\left(\frac{\pi}{2 R}\right)^{2}$.

The proof of Part 3 will result of the application of the iterative method of super and subsolutions (since the comparison principle does not apply directly to solutions of the problem $(D P)$ ). We start by proving that if $\lambda^{\#}$ is the eigenvalue mentioned in Part 2 then we can chose $m^{\#} \in[0,1)$ such that

$$
\lambda^{\#}=\lambda^{*}\left(m^{\#}\right)
$$

with $\lambda^{*}(m)$ the critical eigenvalue of the nonlinear problem $P\left(R, m, V_{0}, \lambda\right)$ given in Proposition 1.5. Indeed, for any $m \in[0,1)$ we have

$$
\lambda^{*}(m)=\frac{\varphi(m)}{2 R^{2}}
$$

where $\varphi(m):=\int_{0}^{r_{F}} \frac{d r}{\sqrt{\frac{r^{m+1}}{m+1}-\frac{r^{2}}{2}}}$, with $r_{F}$ given by (2.5). Obviously function $\varphi(m)$ is continuous, $\varphi(m)>0$ for any $m \in[0,1)$ and

$$
\lim _{m \nearrow 1} \varphi(m)=+\infty .
$$

Moreover, it is not difficult to check that

$$
\int_{a}^{b} \frac{d r}{\sqrt{r-\frac{r^{2}}{2}}}=\sqrt{2}(\arcsin (1-a)-\arcsin (1-b)),
$$

and thus

$$
\varphi(0)=\int_{0}^{2} \frac{d r}{\sqrt{r-\frac{r^{2}}{2}}}=\sqrt{2} \pi .
$$

Then property (2.16) holds since we know that 


$$
\lambda^{\#} \geqslant\left(\frac{\pi}{2 R}\right)^{2}>\frac{\sqrt{2} \pi}{2 R^{2}}=\lambda^{*}(0)
$$

On the other hand the comparison principle holds for solutions of the problem

$$
D P(V, f)\left\{\begin{array}{lr}
-\frac{d^{2} u}{d x^{2}}+V(x) u=f(x) & \text { in } \Omega, \\
u=0 & \text { on } \partial \Omega,
\end{array}\right.
$$

in the sense that, since $\frac{C}{d(x, \partial \Omega)^{2}} \leqslant V(x)$, if $\underline{f}, \bar{f} \in H^{-1}(\Omega)$ and $\underline{f} \leqslant \bar{f}$ in $H^{-1}(\Omega)$ then there exist $\underline{u}, \bar{u} \in H_{0}^{1}(\Omega)$ solutions of $D P(V, \underline{f})$ and $D P(V, \bar{f})$, respectively, such that $\underline{u}(x) \leqslant$ $\bar{u}(x)$ a.e. $x \in \Omega$. The proof of this follows by applying again the Hardy inequality (a different argument, when $f, \bar{f} \in L^{1}(\Omega, \delta)$, can be found in [24]). Then we can apply the iterative method of super and subsolutions. We start by building the supersolution of $D P\left(V, \lambda^{\#}, \Omega\right)$ of the form $\bar{u}(x)=v_{\lambda^{*}\left(m^{*}\right)}\left(x: \bar{V}_{0}\right)$ with $v_{\lambda^{*}\left(m^{\#}\right)}\left(x: \bar{V}_{0}\right)$ the flat solution of $P\left(R, m^{\#}, \bar{V}_{0}, \lambda^{*}\left(m^{\#}\right)\right)$ with $\bar{V}_{0}$ to be chosen later. Thanks to estimates (1.10), (1.11) and assumption (1.7), for any $x \in \bar{\Omega}$ we have

$$
\frac{\bar{V}_{0}}{\left|v_{\lambda^{*}\left(m^{\#}\right)}(x)\right|^{1-m^{\#}}} \leqslant \frac{\bar{V}_{0}}{\left(K^{\#}\right)^{1-m^{\#}}} \frac{1}{d(x, \partial \Omega)^{2}} \leqslant V(x),
$$

if the condition

$$
\frac{\bar{V}_{0}}{\left(K^{\#}\right)^{1-m^{\#}}} \leqslant \underline{C}
$$

holds. As a matter of fact, from the proof of Proposition 1.5 we can see that (2.17) is equivalent to

$$
\frac{1}{\underline{K}^{1-m^{\#}}} \leqslant \underline{C}
$$

where $\underline{K}$ is the bound associated to the "direct case" $\bar{V}_{0}=1$ and $\lambda=1$ (see Remark 2.3).Then, if we assume (2.18) we have

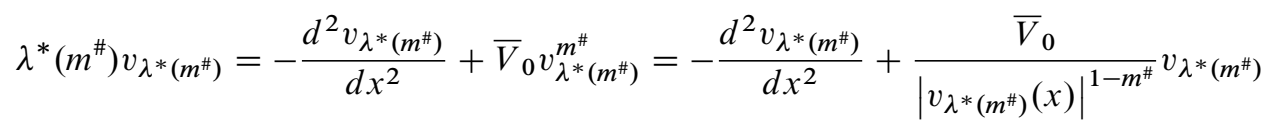

$$
\begin{aligned}
& \leqslant-\frac{d^{2} v_{\lambda^{*}\left(m^{\#}\right)}}{d x^{2}}+\frac{\bar{V}_{0}}{\left(K^{\#}\right)^{1-m^{\#}}} \frac{v_{\lambda^{*}\left(m^{*}\right)}}{d(x, \partial \Omega)^{2}} \leqslant-\frac{d^{2} v_{\lambda^{*}\left(m^{*}\right)}}{d x^{2}}+V(x) v_{\lambda^{*}\left(m^{\#}\right)},
\end{aligned}
$$

which proves that $v_{\lambda^{*}\left(m^{\#}\right)}\left(x: \bar{V}_{0}\right)$ is a supersolution (notice that for the moment $\bar{V}_{0}$ is arbitrary).

The construction of a subsolution is more delicate. In fact we shall built a continuum of subsolutions. Given $\widehat{V}_{0}>0$ (to be chosen later) we shall take a suitable $\lambda>\lambda^{*}\left(m^{\#}\right)$ and $\underline{u}(x)=v_{\lambda}\left(x: \widehat{V}_{0}\right)$ solution of $P\left(R, m^{\#}, \widehat{V}_{0}, \lambda\right)$. By properties d) mentioned in the proof of Proposition 1.5 , if $\lambda=\lambda^{*}\left(m^{\#}: \widehat{V}_{0}\right) \omega$ with $\omega>1$ we have a family $S_{1}(\lambda)$ of compact support nonnegative solutions with connected support defined by

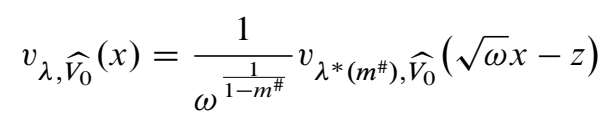


where the shifting argument $z$ is arbitrary among the points $z \in(-R, R)$ such that support $v_{\lambda, \widehat{V_{0}}}(\cdot) \subset(-R, R)$. Then, arguing as in the case of the supersolution, we have

$$
-\frac{d^{2} \underline{u}}{d x^{2}}+V(x) \leqslant-\frac{d^{2} \underline{u}}{d x^{2}}+\widehat{V}_{0} \underline{u}^{m^{\#}}-\left(\lambda-\lambda^{*}\left(m^{\#}\right)\right) \underline{u}=\lambda^{*}\left(m^{\#}\right) \underline{u}
$$

assumed

$$
\bar{C} \leqslant \frac{\omega}{\left(\bar{K}^{\#}\right)^{1-m^{\#}}}-\left(\lambda-\lambda^{*}\left(m^{\#}\right)\right) R^{2},
$$

since we have

$$
\left(\frac{\omega}{(\bar{K})^{1-m^{\#}}}-\lambda^{*}\left(m^{\#}\right)(\omega-1) R^{2}\right) \frac{1}{d(x, \partial \Omega)^{2}} \leqslant \widehat{V}_{0} \underline{u}^{m^{\#}-1}-\left(\lambda-\lambda^{*}\left(m^{\#}\right)\right),
$$

with $\bar{K}$ the upper bound associated to the "direct case" $\widehat{V}_{0}=1$ and $\lambda=1$ (see Remark 2.3). If we define $\varepsilon:=\lambda-\lambda^{*}\left(m^{\#}\right)$ then

$$
\omega=\frac{\lambda}{\lambda^{*}\left(m^{\#}\right)}=\frac{\lambda^{*}\left(m^{\#}\right)+\varepsilon}{\lambda^{*}\left(m^{\#}\right)}
$$

and condition (2.20) can be written as

$$
\bar{C} \leqslant \frac{\frac{\lambda^{*}\left(m^{\#}\right)+\varepsilon}{\lambda^{*}\left(m^{\#}\right)}-\varepsilon R^{2}(\bar{K})^{1-m^{\#}}}{(\bar{K})^{1-m^{\#}}}=\frac{1+\varepsilon\left(\frac{1}{\lambda^{*}\left(m^{\#}\right)}-R^{2}(\bar{K})^{1-m^{\#}}\right)}{(\bar{K})^{1-m^{\#}}} .
$$

This implies that $\underline{u}$ is a subsolution.

Finally, to apply the super and subsolution method we must check that

$$
\underline{u}(x) \leqslant \bar{u}(x) \text { for any } \quad x \in \Omega .
$$

From the definitions of $\underline{u}(x)$ and $\bar{u}(x)$ we have that (2.21) holds if

$$
\frac{\widehat{V}_{0}}{\lambda} \leqslant \frac{\bar{V}_{0}}{\lambda^{*}\left(m^{\#}\right)},
$$

or equivalently

$$
\frac{\lambda^{*}\left(m^{\#}\right)+\varepsilon}{\lambda^{*}\left(m^{\#}\right)} \geqslant \frac{\widehat{V}_{0}}{\bar{V}_{0}} .
$$

Thus we can proceed as follows; we assume

$$
\bar{C}<\frac{1}{(\bar{K})^{1-m^{\#}}}
$$

Then, if $\frac{1}{\lambda^{*}\left(m^{*}\right)}-R^{2}(\bar{K})^{1-m^{*}} \geqslant 0$ we can take $\varepsilon>0$ arbitrary and then $\bar{V}_{0}$ and $\widehat{V}_{0}$ such that (2.23) holds. If by the contrary $\frac{1}{\lambda^{*}\left(m^{\#}\right)}-R^{2}(\bar{K})^{1-m^{*}}<0$ then we take

$$
\varepsilon<\frac{1-\bar{C}(\bar{K})^{1-m^{\#}}}{\left(R^{2}(\bar{K})^{1-m^{\#}}-\frac{1}{\lambda^{*}\left(m^{\#}\right)}\right)}
$$


and again $\bar{V}_{0}$ and $\widehat{V}_{0}$ such that (2.23) holds.

Then, by the super and subsolution method, we get the existence of a minimal $\underline{u}^{*}(x)$ and maximal $\bar{u}^{*}(x)$ solution of $(D P)$ such that

$$
\underline{u}(x) \leqslant \underline{u}^{*}(x) \leqslant \bar{u}^{*}(x) \leqslant \bar{u}(x) \quad \text { for any } \quad x \in \Omega .
$$

Since there is a continuum of subsolutions we can shift them in order to get that $\bar{u}^{*}(x)>0$ for any $x \in \Omega$. Moreover from the spectral theory necessarily $\Lambda \underline{u}^{*}(x)=u^{\#}=\bar{\Lambda} \bar{u}^{*}(x)$ for some $\underline{\Lambda}, \bar{\Lambda}>0$ and the estimates (1.9) hold for the solutions of the linear problem thanks to Proposition 1.5 .

Proof of Corollary 1.7. As it is shown in [20], the nodal solutions $v_{\lambda_{n}^{*}(m)}$ of the semilinear problem $P\left(R, m, V_{0}, \lambda\right)$ corresponding to suitable values $\lambda_{n}^{*}(m)$ of the parameter $\lambda$ bifurcating at the infinity from the simple eigenvalues $\lambda_{n}, n \in \mathbb{N}$, are obtained by rescaling, gluing and translating the unique positive flat solution corresponding to $\lambda^{*}(m)$. Thus the conclusion is an obvious consequence of Proposition 1.5.

REMARK 2.4 Theorem 1.2 and Proposition 1.5 also hold to $N>1$ for suitable convex regular domains, for instance, satisfying the interior sphere condition (see [16]).

Proof of Theorem 1.9. Now the comparison principle can be applied directly and so it suffices to follow the same scheme of proof as in Theorem 1.2. The sub and supersolutions are obtained by solving the associate sublinear problem

$$
\left\{\begin{array}{l}
-\frac{d^{2} v}{d x^{2}}+V_{0} v^{m}=f(x) \quad \text { in }(-R, R), \\
v( \pm R)=0
\end{array}\right.
$$

for suitable choices of $m \in(0,1)$ and $V_{0}>0$. The boundary estimate similar to the given in (1.10) was obtained in Theorem 1.15 of [14] (see also [2]) thanks to the crucial assumption (1.14).

REMARK 2.5 A different type of localizing results concerning the non-linear Schrödinger equation, arising in nonlinear optics,

$$
\mathbf{i} \hbar \frac{\partial \psi}{\partial t}=-\frac{\hbar^{2}}{2 m} \Delta \psi+\boldsymbol{a}|\psi|^{\sigma} \psi, \text { in }(0, \infty) \times \mathbb{R}^{N},
$$

were also presented in the series of lectures [15]. We recall that in most of the papers in the literature it is assumed $\sigma=2$, nevertheless there are many applications in which $\sigma \in(-1,0)$. In a series of papers in collaboration with P. Bégout ([4], [5], [6], [7] and [8]) we prove precise estimates on the location of the support of $\psi(x, t)$, whose boundary gives rise to a free boundary associated to the problem. The techniques of proof are some extensions of the ones of [1] and are entirely different to the ones used in the present paper.

REMARK 2.6 As it will be presented in [17], some of the ideas of this paper can be adapted to the study the existence of "large solutions" of the same type of linear equation

$$
\left\{\begin{array}{lr}
-\Delta u+V(x) u=f(x) & \text { in } \Omega, \\
u=+\infty & \text { on } \partial \Omega,
\end{array}\right.
$$


when the potential $V$ satisfies (1.7). We recall (Theorem 2.10 of [24]) that given $f \in L^{1}(\Omega: \delta$ ), $f(x) \geqslant 0$ a.e. $x \in \Omega, V_{0}>0$, the existence of a large solution of the semilinear problem

$$
\left\{\begin{array}{lr}
-\Delta v+V_{0} v^{m}=f(x) & \text { in } \Omega, \\
v=+\infty & \text { on } \partial \Omega,
\end{array}\right.
$$

requires now the key assumption $m>1$ (compare this condition to the assumption $m \in(0,1)$ used in the proof of Theorem 1.9 for the existence of a flat solution).

Acknowledgments. The author thanks Th. Cazenave, J. Hernández and the anonymous referees for several useful suggestions on previous versions of this paper. The research of the author was partially supported by the project ref. MTM2011-26119 and MTM2014-57113-P of the DGISPI (Spain), the UCM Research Group MOMAT (Ref. 910480) and the ITN FIRST of the Seventh Framework Program of the European Community's (grant agreement number 238702).

\section{REFERENCES}

1. Antontsev, S. Díaz, J. I. \& Shmarev, S., Energy methods for free boundary problems. Applications to nonlinear PDEs and Fluid Mechanics, Series Progress in Nonlinear Differential Equations and Their Applications, No. 48, Birkäuser, Boston, 2002. Zbl0988. 35002 MR1858749

2. Alvarez,L. \& DíAZ, J. I., On the retention of the interfaces in some elliptic and parabolic nonlinear problems, Discrete and Continuum Dynamical Systems 25 (2009), 1-17. Zbl1171. 35368 MR2525165

3. Bandle, C. \& Pozio, M. A., Sublinear elliptic problems with a Hardy potential. Nonlinear Anal., Theory Methods Appl., Ser. A, Theory Methods 119 (Article ID 10319) (2015), 149-166. Zb106446449 MR3334180

4. BÉGOUt, P. \& DiAZ, J. I., On a nonlinear Schrödinger equation with a localizing effect. Comptes Rendus Acad. Sci. Paris 342 Serie I (2006), 459-463. Zb11094. 35113 MR2214595

5. BÉgout, P. \& DiaZ, J. I., Localizing Estimates of the Support of Solutions of Some Nonlinear Schrödinger Equations: The Stationary Case. Annales de l'Institut Henri Poincare (C) Non Linear Analysis 29 (2012), 35-58. Zbl1241. 35185 MR2876246

6. BÉGout, P. \& DiAZ, J. I., A sharper energy method for the localization of the support to some stationary Schrödinger equations with a singular nonlinearity. Discrete and Continuous Dynamical System Series A 34 (2014), 3371-3382. Zbl1305.35022 MR3190983

7. BÉGOUT, P. \& DiAZ, J. I., Self-similar solutions with compactly supported profile of some nonlinear Schrödinger equations. Electronic Journal Differential Equations 2014 (2014), 1-15. Zbl1291. 35331 MR3193996

8. BÉGOUT, P. \& DiAZ, J. I., Existence of weak solutions to some stationary Schrödinger equations with singular nonlinearity. Rev. R. Acad. Cienc. Exactas Fís. Nat., Ser. A Mat. RACSAM 109 (2015), 43-63. Zb11309.35126 MR3315701

9. Bertsch, M. \& Rostamian, R., The principle of linearized stability for a class of degenerate diffusion equations. J. Differ. Equat 57 (1985) 373-405. Zb10583. 35061 MR0790282

10. Benilan, Ph., Brezis, H. \& Crandall, M. G., A semilinear equation in $\mathrm{L}^{1}\left(\mathrm{R}^{N}\right)$, Ann. Scuola Norm. Sup. Pisa 4 (1975), 523-555. Zbl0314. 35077 MR0390473

11. Brezis, H., Solutions of variational inequalities with compact support. Uspekhi Mat. Nauk., 129 (1974) 103-108. Zb10304.35036 MR0481460

12. BRezis, H. \& StAmpaCchia, G., Une nouvelle méthode pour l'étude d'écoulements stationnaires. C.R. Acad. Sci. 276 (1973), 129-132. Zb10246. 35021 MR0315973 
13. BRezis, H. \& Stampacchia, G., The hodograph method in Fluid-Dynamics in the light of Variational Inequalities. Arch. Rat. Mech.Anal. 61 (1976), 1-18. Zbl0334. 76004 Mr0477505

14. DíAZ, J. I.. Nonlinear Partial Differential Equations and Free Boundaries. Research Notes in Mathematics, 106, Pitman, London 1985. Zb10595. 35100 MR0853732

15. DÍAZ, J. I., Lectures on the Schrödinger equation for the "infinite well potential. In the books of abstracts of the congresses Quasilinear PDEs, Tours (France), June 4-6, 2012; Nonlinear Partial Differential Equations, Valencia (Spain) July 1-5, 2013; Free Boundary Problems Programme of the Newton Institute (January-July 2014), Cambridge (UK), May 22, 2014.

16. DíAZ, J. I., On the ambiguous treatment of the Schrödinger equation for infinite potential well and an alternative via flat solutions: the multi-dimensional case. To appear.

17. DíAZ, J. I., On the existence of large solutions for linear elliptic equations with a Hardy absorption potential. To appear.

18. DíAZ, J. I. \& HERnÁndeZ, J., Global bifurcation and continua of nonnegative solutions for a quasilinear elliptic problem. Comptes Rendus Acad. Sci. Paris 329 Série I (1999), 587-592. Zb10937.35064 MR1717114

19. DÍAZ, J. I. \& HERNÁNDEZ. J., On a countable set of branches bifurcating from the infinity of nodal solutions for a singular semilinear equation. Proc. of the 5th International Conference on Approximation Methods and Numerical Modelling in Environment and Natural Resources, MAMERN13, .B. Amaziane et al. eds., Editorial Universidad de Granada, Granada, Spain, 2013, p. 118 (see also the extended abstract version at https://www.ugr.es/ mamern/mamern13)

20. Díaz, J. I. \& Hernández, J., Positive and nodal solutions bifurcating from the infinity for a semilinear equation: Solutions with compact support. Portugaliae Mathematica 72 (2015), 145-160.

21. DíAz, J. I., HeRnándeZ, J. \& MAAGLi, H., to appear.

22. Díaz, J. I., Hernández, J, \& MANCEBo, F. J., Branches of positive and free boundary solutions for some singular quasilinear elliptic problems. J. Math. Anal. Appl. 352 (2009) 449-474). Zbl1173. 35055 MR2499916

23. Díaz, J. I., Hernández, J. \& Ilyasov, Y., On the existence of positive solutions and solutions with compact support for a spectral nonlinear elliptic problem with strong absorption. Nonlinear Anal., Theory Methods Appl., Ser. A, Theory Methods 119 (Article ID 10437) (2015), 484-500. Zbl 06446470 MR3334201

24. Díaz, J. I. \& RAkotoson, J.-M., On very weak solutions of semilinear elliptic equations with right hand side data integrable with respect to the distance to the boundary, Discrete and Continuum Dynamical Systems. 27, (2010), 1037-1058. Zbl1198. 35095 MR2629573

25. Galindo, A. \& PAScual, P., Quantum Mechanics I, Springer-Verlag, Berlin, 1990. Zb10824.00008 MR1079543

26. Gamow, G., Zur Quantentheorie des Atomkernes, Zeitschrift für Physik 51 (1928), 204-212.

27. Hernandez, J., Mancebo, F.J. \& Vega, J.M., On the linearization of some singular nonlinear elliptic problems and applications. Ann. Inst. H. Poincaré Anal. 19 (2002), 777-813. Zbl1020 . 35065 MR1939086

28. Joglekar, Y. N., Particle in a box with a function potential: Strong and weak coupling limits. Am. J. Phys. 77 (2009), 734-736.

29. KAPER, H. \& KWOng, M., Free boundary problems for Emden-Fowler equation. Differential and Integral Equations 3 (1990), 353-362. Zb10726. 34024 MR1025184

30. Leighton, R. B., Principles of Modern Physics. McGraw-Hill Inc. New York, 1959.

31. NAIMARK, M. A. Linear Differential Operators, Ungr, New York, 1968. Zb10227. 34020 Mr0262880

32. Protter, M. H. \& Weinberger, H. F., Maximum Principles in Differential Equations. New York, Springer-Verlag, 1984. Zb10549.35002 MR0762825

33. PUCCI, P. \& SERrin, J. The maximum principle, Progress in Nonlinear Differential Equations and Their Applications, 73, Birkhäuser Verlag, Basel, 2007. Zbl1134. 35001 MR2356201 
34. Strauss, W., Partial Differential Equations. An Introduction, John Wiley and Sons, New York, 1992. Zb10817.35001 MR1159712

35. VÁzquez, J. V. A strong maximum principle for some quasilinear elliptic equations, Appl. Math. Optim. 12 (1984), 191-202. Zbl0561. 35003 MR0768629 